\title{
Curative resection by hybrid 2-port HALS in a patient with advanced cecal cancer invading the urinary bladder: A case report
}

\author{
MASAYA MUKAI ${ }^{1}$, KYOKO KISHIMA ${ }^{1}$, SHIN-ICHI IIZUKA ${ }^{1}$, HIROSHI FUKUMITSU ${ }^{1}$, MAKI FUKASAWA ${ }^{1}$, \\ NAOKI YAZAWA ${ }^{1}$, TAKAYUKI TAJIMA ${ }^{1}$, MASATO NAKAMURA ${ }^{2}$ and HIROYASU MAKUUCHI ${ }^{3}$ \\ ${ }^{1}$ Department of Surgery, Tokai University Hachioji Hospital, Ishikawa-cho 1838, Hachioji, Tokyo 192-0032; Departments \\ of ${ }^{2}$ Pathology and ${ }^{3}$ Surgery, Tokai University School of Medicine, Bohseidai, Isehara, Kanagawa 259-1193, Japan
}

Received November 25, 2008; Accepted March 6, 2009

DOI: $10.3892 /$ or_00000365

\begin{abstract}
A 67-year-old woman was referred to our department for assessment of a tumor in the right lower abdomen. Advanced cecal cancer invading the urinary bladder was diagnosed, and laparoscopy assisted colorectal surgery (LACS)-hybrid 2-port hand-assisted laparoscopic surgery (HALS) was performed in February 2008. Intraoperative laparoscopic observation revealed direct invasion of the urinary bladder by the primary tumor, so an approximately 6-cm transverse suprapubic incision was made. Under direct vision through this incision, full-thickness partial cystectomy was performed to remove the tumor invading the bladder. Then D3 right hemicolectomy was performed under pneumoperitoneum. In this patient with advanced cecal cancer invading the bladder, we performed radical curative surgery by hybrid 2-port HALS, a minimally invasive procedure in which a 6-cm incision was made in addition to the hand access site and favorable results were obtained.
\end{abstract}

\section{Introduction}

In recent years, minimally invasive laparoscopy assisted colorectal surgery (LACS) has become very popular, and the indications for this type of operation have increased dramatically, ranging from additional bowel resection for stage I colorectal cancer to curative surgery for stage II/III

Correspondence to: Dr Masaya Mukai, Department of Surgery, Tokai University Hachioji Hospital, Ishikawa-cho 1838, Hachioji, Tokyo 192-0032, Japan

E-mail:mukai.masaya@hachioji-hosp.tokai.ac.jp

Abbreviations: HALS, hand assisted laparoscopic surgery; LACS, laparoscopy assisted colorectal surgery

Key words: colorectal cancer, hybrid HALS, laparoscopy assisted colorectal surgery, hand assisted laparoscopic surgery, cecal cancer cancer and palliative operations for stage IV cancer (1-5). In Western countries, LACS is performed as hand-assisted laparoscopic surgery (HALS) and/or hybrid HALS combined with manipulation under direct vision (6-9). In contrast, pure LACS is more popular in Japan, which requires 5-6 ports including a camera port and a small $4-5 \mathrm{~cm}$ incision $(10,11)$. However, 4 clamps are used by the surgeons and the first assistant for pure LACS, so at least 3 skilled surgeons who are also experts in camera manipulation are necessary. Compared with standard open laparotomy, pure LACS has the following disadvantages: i) lack of tactile sensation, ii) limitations on surgical manipulation of large tumors, iii) difficulty in assessing the entire surgical field, iv) a long operating time because of endoscopic manipulation and v) difficulting in acquiring the necessary skills because of the system of technical authorization in Japan. To overcome these problems, we use hybrid 2-port HALS as the standard surgical technique, in which a $5-6 \mathrm{~cm}$ incision is made first for hand access $(5,12)$.

In a patient with advanced cecal cancer and direct invasion of the urinary bladder, a large median abdominal incision was avoided by minimally invasive surgery that involved hybrid 2-port HALS with a 6-cm suprapubic incision in addition to a $6-\mathrm{cm}$ longitudinal midline incision in the upper abdomen for hand access. We obtained favorable results, as reported here.

\section{Case report}

The patient was a 67 -year-old woman. She was previously healthy and the past history was not contributory. From around August 2007, she noted dull pain in the lower right abdomen, but did not seek treatment. In December 2007, a mass the size of a hen's egg was detected in the lower right quadrant of the abdomen. She was then referred to our hospital and admitted for detailed assessment of the tumor. Barium enema and colonoscopy revealed a circumferential tumor with a longest diameter of $\sim 6 \mathrm{~cm}$ below the Bauhin's valve in the cecum, and a diagnosis of well-differentiated adenocarcinoma was made by tumor biopsy (Fig. 1, Left). Before surgery, imaging studies such as CT and MRI strongly 


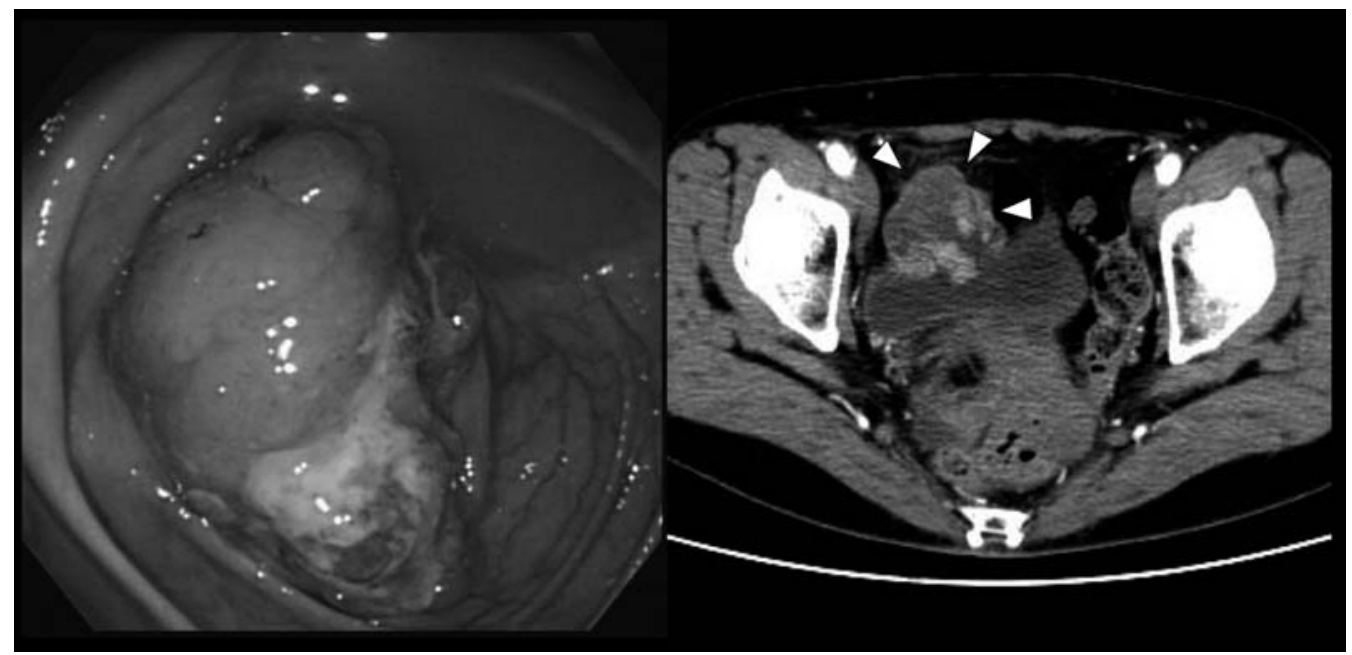

Figure 1. Colonoscopy reveals a circumferential tumor $\sim 6 \mathrm{~cm}$ in longer diameter below the Bauhin's valve in the cecum. Well-differentiated adenocarcinoma was diagnosed by biopsy (Left). Preoperative CT finding reveals direct invasion of the right lateral wall and vertex of the urinary bladder by the cecal cancer (Right, arrowheads).

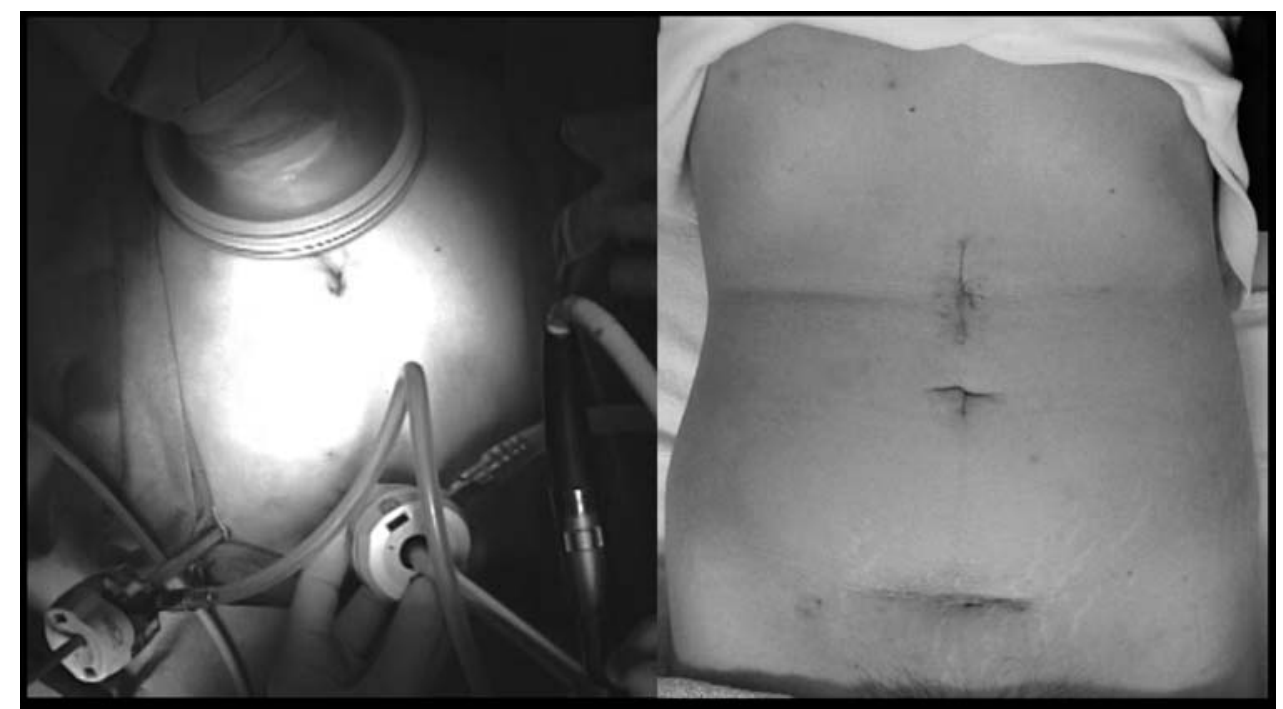

Figure 2. Photograph showing the abdomen at the start of surgery by hybrid 2-port HALS (Left). After attaching a hand access device (Lapdisc ${ }^{\circledR}$, regular type, $120 \times 120 \mathrm{~mm}$ ) to the $\sim 6-\mathrm{cm}$ midline incision in the upper abdomen, 5 - and 12-mm ports for surgical manipulation and a camera were respectively inserted into the suprapubic region (Left). Photograph of the abdomen at about 12 months after hybrid 2-port HALS (Right).

suggested direct invasion of the right lateral wall and the vertex of the urinary bladder, but the patient had no symptoms of micturition disorders, such as cystitis or pollakiuria (Fig. 1; Right, arrowheads). After informed consent for LACS-HALS and standard median laparotomy, was obtained from the patient and her family, LACS-hybrid 2-port HALS was performed in February 2008 (Fig. 2, Left).

Operative findings for hybrid 2-port HALS. After a device (Lapdisc ${ }^{\circledR}$, regular type, 120x120 mm, Hakkou Co., Ltd., Nagano, Japan) was attached to the $\sim 6-\mathrm{cm}$ median longitudinal incision that was initially made in the upper abdomen as a hand access site, laparoscopic observation was performed under pneumoperitoneum. This revealed that the primary tumor, which was a palpable mass $\sim 6 \mathrm{~cm}$ in longer diameter in the cecum, showed direct invasion of the right lateral wall of bladder over an area $\sim 4-5 \mathrm{~cm}$ extending toward the vertex (Fig. 3A). Since the 12-mm camera port was located at the upper border of the pubis, a 6-cm transverse incision that included this port was made to provide access to the urinary bladder. Under direct vision through the mini-laparotomy incision, the vertex of the bladder including the site of tumor invasion was resected with a wide safety margin (Fig. 3B). After it was confirmed by intraoperative frozen section examination that the margin was negative for cancer invasion, the bladder was closed in 2 layers (Fig. 3C). Subsequently, D3right hemicolectomy was performed, and complete closure of the mesentery of the terminal ileum and transverse mesocolon was followed by reconstruction with functional end-to-end anastomosis (Fig. 3D). After hemostasis was confirmed, the peritoneal cavity was irrigated and a drain tube was inserted through the 5-mm port. Then the hand access site and the 


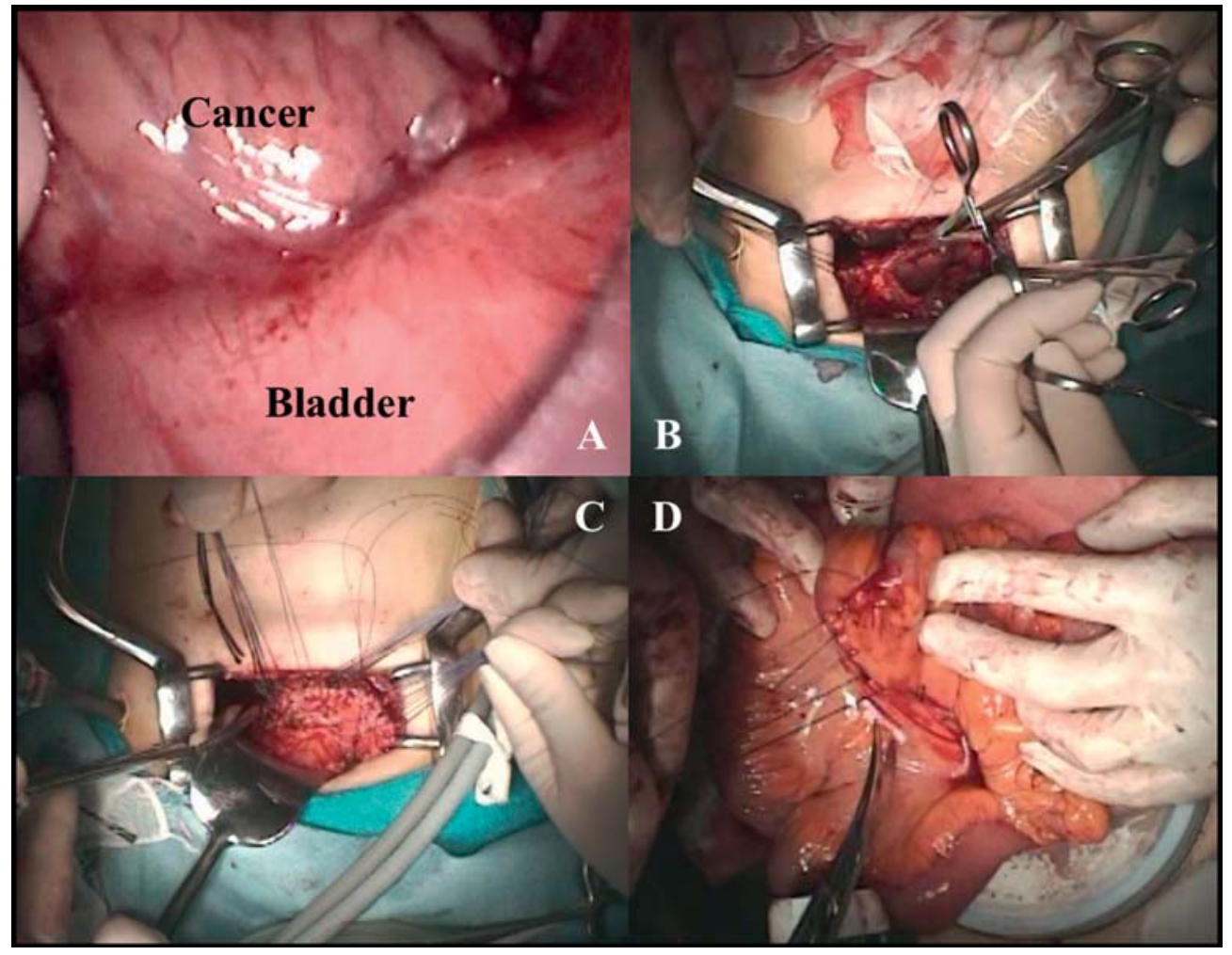

Figure 3. Intraoperative photograph taken during hybrid 2-port HALS. Cecal cancer is invading the right lateral wall of the bladder (A). An $\sim 6$-cm transverse suprapubic incision incorporating the camera port was made, and the tumor invading the bladder was removed by partial cystectomy under direct vision (B). After it was confirmed by intraoperative frozen section examination that the resection margin was negative for cancer invasion, the wall of the bladder was closed in 2 layers under direct vision (C). After D3-right hemicolectomy was performed, closure of the mesentery of the terminal ileum and the transverse mesocolon was followed by reconstruction employing extracorporeal functional end-to-end anastomosis (D).

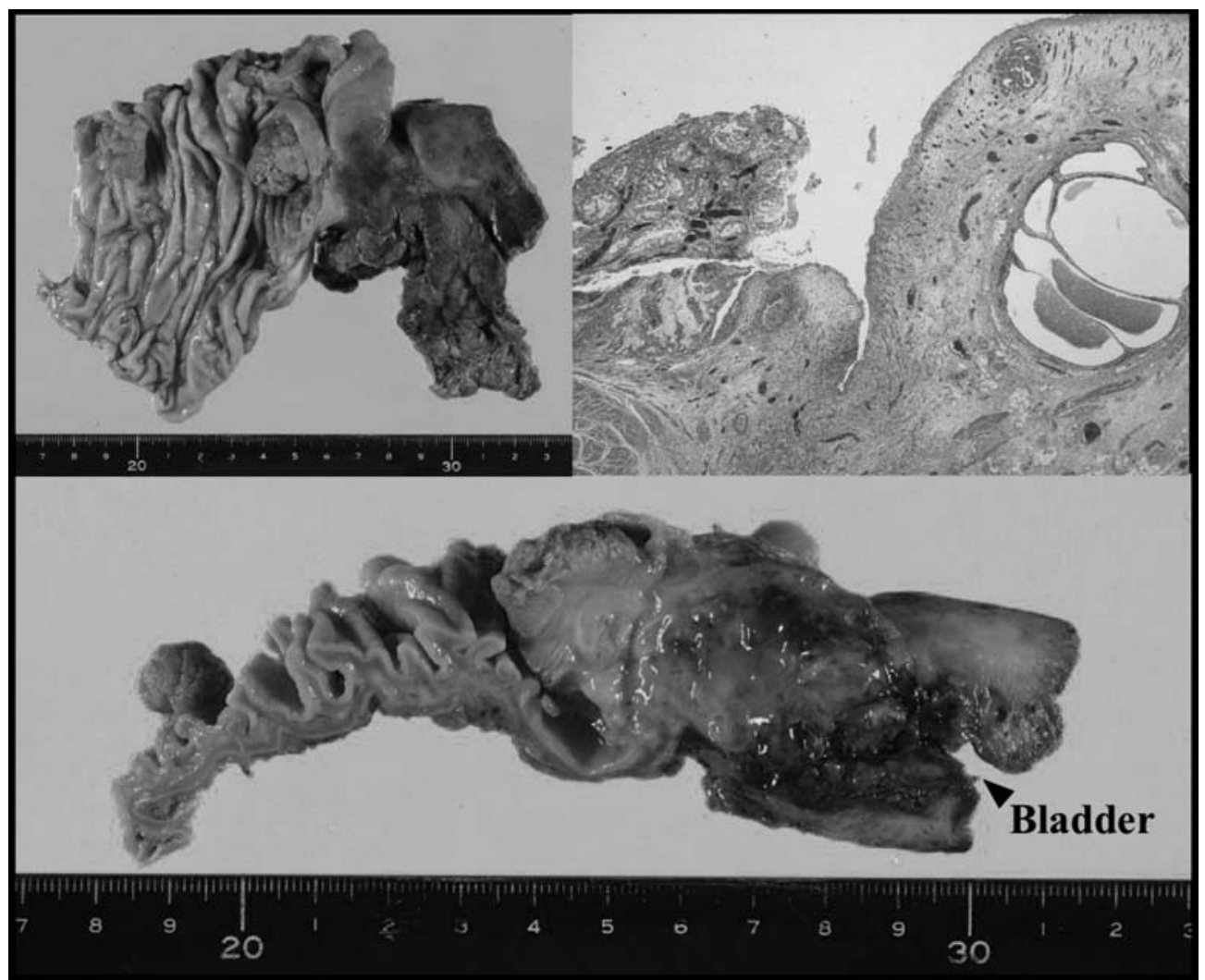

Figure 4. The tumor was an advanced cecal cancer $\sim 6 \mathrm{~cm}$ in longer diameter. The lesion was POHOLNO (0/25 nodes) and SEI (serosal exposure and infiltration to the urinary bladder). The tumor was a well-differentiated adenocarcinoma and was pathologically in stage II, although there was deep invasion of an area $3 \mathrm{~cm}$ in diameter in the wall of the bladder. 
transverse suprapubic incision were closed, and the operation was completed.

The tumor was an advanced cecal cancer that was $6 \mathrm{~cm}$ in longer diameter and POHONOSEI (serosal exposure and infiltration to the urinary bladder). There were no involved lymph nodes $(0 / 25)$ and the tumor was pathologically in stage II, although pathological examination revealed invasion deep into the wall of the bladder over an area $3 \mathrm{~cm}$ in diameter (Fig. 4). There were no postoperative complications, including micturition disorders, and the patient was discharged from hospital at 13 days postoperatively. She has been treated for 12 months postoperatively with oral chemotherapy on an outpatient basis, and there have been no signs of tumor recurrence/metastasis (Fig. 2, Right).

\section{Discussion}

In Japan, pure LACS has reached a high technical level and has become widespread. However, because several skilled surgeons are required to reduce to operating time and because there has been a shortage of anesthesiologists, it cannot be easily performed at some relatively large general hospitals and there are some hospitals that have abandoned routine LACS. Considering these problems, hybrid 2-port HALS has been adopted at our department as the standard technique since July 2007, and it has been performed in more than 75 patients $(5,12)$. This technique has the following advantages: i) the hand can be used safely under magnification and monitoring; ii) because of full grasping with tactile sensation, even patients with large tumors can be operated on smoothly and less invasively; iii) only two surgeons are needed; iv) the operating time is shorter because this procedure is an extension of standard laparotomy; and v) less time is required to acquire the necessary skills. Hybrid 2-port HALS also has advantages over pure LACS with respect to surgical manipulation. In addition to anastomosis, complete closure of the colorectal mesentery after colorectal resection, ligation of blood vessels, and dissection of lymph nodes can be performed relatively easily under direct vision, and this technique may be safely applied in patients with ileus and patients with cancer invading other organs such as our case $(5,12,13)$. As complications, conversion to open laparotomy and suture failure were not noted in the patients having colon surgery. Mild stenosis at the anastomosis was only noted in 1 patient, while wound infection occurred in 4/20 patients early after surgery. However, re-operation due to postoperative hemorrhage, delayed small bowel perforation or ureteric injury was not required (data not shown).

In recent years, the range of indications for LACS in Japan has increased markedly to include stage I-IIIa (N1 lymph node metastasis to $\leq 3$ nodes) to stage IV palliative surgery, although this depends on the criteria including stage IIIb (N2 metastasis) used by each institution (1-5). In Western countries, LACS is often combined with radiation therapy for the treatment of advanced low rectal cancer (14-16). In Japan, N3 dissection of the lateral lymph nodes around the internal and external iliac arteries/veins has long been investigated rather than radiation therapy for rectal cancer (17-19). It has been reported by many researchers that the outcome was more favorable when the lateral lymph nodes were removed in patients with lateral lymph node metastasis. However, there is still controversy about prophylactic bilateral lateral lymph node dissection. Therefore, we consider that LACS is indicated for rectal cancer patients with no evidence of lateral lymph node metastasis before surgery $(5,12)$. When hybrid 2-port HALS is performed as an elective surgery, there is no clear difference from laparotomy with respect to dissection of layers if the left hand can reach the distal side of a tumor. Moreover, surgical manipulation in the pelvic floor or other difficult sites, which is usually performed almost blindly, can be performed safely under magnification and monitoring. Therefore, LACS-hybrid 2-port HALS may be applicable to a wide variety of patients. At our department, the indications for LACS-hybrid 2-port HALS are as follows: i) patients who can tolerate general anesthesia for approximately $2 \mathrm{~h}$, ii) patients with no severe concomitant diseases, such as cardiac/pulmonary disease, and iii) patients with a P.S. of $0-1$ aged $\leq 75$ years if possible. It has also been reported that this technique is indicated for rectal cancer patients without obvious lateral lymph node metastasis $(5,12)$. Although a $6-\mathrm{cm}$ transverse suprapubic incision is standard in patients with advanced lower rectal cancer, if change to small incision for hand access such as a median longitudinal incision or a pararectal incision in the lower abdomen, was made near the site of metastatic lymph nodes, lateral lymph node dissection under direct vision could also be performed adequately by hand access site. In the future, it will be necessary to perform a detailed comparison with standard laparotomy to assess recurrence such as local recurrence in the pelvic cavity and distant metastasis in a larger series of patients, including those with advanced low rectal cancer.

\section{Acknowledgements}

This study was supported by grants from the Occult Neoplastic Cells Research and Study Group (No. 2008-5007; Tokai University Hachioji Hospital, Tokyo, Japan) and the Research and Study Program of Tokai University Educational System General Research Organization (No. 2007-04; Tokai University School of Medicine, Kanagawa, Japan).

\section{References}

1. Mukai M, Tokunaga N, Ishida H, Makuuchi H, Tajima $\mathrm{T}$ and Mitomi T: Clinical experiences with laparoscopic colectomy. Dig Endosc 9: 11-15, 1997.

2. Mukai M, Okamoto Y, Oida Y, Mukoyama S, Ito I, Nakasaki H, Kawai K, Sato S and Makuuchi H: Endoscopic mucosal resection for superficially spreading colonic neoplasms larger than $5 \mathrm{~cm}$ in the right colon after dilute sodium hyaluronate injection: report of two cases. Endoscopy 35: 973-975, 2003.

3. Makuuchi M and Sugihara K (ed): Knacks \& Pit falls: Surgery of the Colon, Rectum and Anus. 2nd edition. Bunkoudou, Co., Ltd., Tokyo, 2004.

4. Guide Line of Large Bowel Cancer in Japan. Japanese Society for Cancer of the Colon and Rectum, Tokyo, 2005.

5. Mukai M, Tanaka A, Tajima T, Yamagiwa T, Okada K, Fukasawa M, Sato K, Oida Y, Nakamura M and Makuuchi H: Two-port hand-assisted laparoscopic surgery (HALS) in 2-stage treatment for complete obstruction by left colon cancer: A case report. Oncol Rep 19: 875-879, 2008.

6. Romanelli JR, Kelly JJ and Litwin DE: Hand-assisted laparoscopic surgery in United States: an overview. Semin Laparosc Surg 8: 96-103, 2001.

7. Nakajima K, Lee SW, Cocilovo C, Foglia C, Kim K, Sonoda T and Milson JW: Hand-assisted laparoscopic colorectal surgery using GelPort. Surg Endosc 18: 102-105, 2004. 
8. Nakajima K, Lee SW, Cocilovo C, Foglia C, Sonoda T and Milson JW: Laparoscopic total colectomy: hand-assisted vs. standard technique. Surg Endosc 18: 582-586, 2004.

9. Yamaguchi Y, Minami K, Kawabuchi Y, Emi M and Toge T: Anterior resection of rectal cancer through a one hand-size incision with or without laparoscopy: proposal of one hand-size incision surgery (OHaSIS). J Surg Res 129: 136-141, 2005.

10. Jacobs M, Verdja JC and Goldstein HS: Minimally invasive colon resection (laparoscopic colectomy). Surg Laparosc Endosc 1: 144-150, 1991

11. The Clinical Outcomes of Surgical Therapy Study Group: A comparison of laparoscopically assisted and open colectomy for colon cancer. N Engl J Med 350: 2050-2059, 2004.

12. Mukai M, Fukasawa M, Kishima K, Iizuka S, Fukumitsu H, Yazawa N, Tajima T, Nakamura M and Makuuchi H: Transanal reinforcing sutures after double stapling for low rectal cancer: Report of two cases. Oncol Rep 21: 335-339, 2009.

13. Mukai M, Himeno S, Mukoyama S, Tajima T, Saito Y, Ito I, Nakasaki H, Sato S and Makuuchi $\mathrm{H}$ : Is the temporally loop colostomy in the right transverse colon appropriate for complete obstruction by colorectal cancer? Oncol Rep 10: 693698, 2003.

14. NIH Consensus Conference: Adjuvant therapy for patients with colon and rectal cancer. JAMA 264: 1444-1450, 1990.
15. Krook JE, Moertel CG, Gunderson LL, Wieand HS, Collins RT, Beart RW, Kubista TP, Poon MA, Meyers WC, Mailliard JA, Twito DI, Morton RF, Veeder MH, Witzig TE, Cha S and Vidyarthi SC: Effective surgical adjuvant therapy for high-risk rectal carcinoma. N Engl J Med 324: 709-715, 1991.

16. Wolmark N, Wieand HS, Hyams DM, Colangelo L, Dimitrov NV, Romond EH, Wexler M, Prager D, Cruz AB Jr, Gordon PH, Petrelli NJ, Deutsch M, Mamounas E, Wickerham DL, Fisher ER, Rockette $\mathrm{H}$ and Fisher B: Randomized trial of postoperative adjuvant chemotherapy with or without radiotherapy for carcinoma of the rectum: National Surgical Adjuvant Breast and Bowel Project Protocol R-02. J Natl Cancer Inst 92: 388-396, 2000.

17. Moreira LF, Hizuta A, Iwagaki H, Tanaka N and Orita K: Lateral lymph node dissection for rectal carcinoma below the peritoneal reflection. Br J Surg 81: 293-296, 1994.

18. Hida J, Yasutomi M, Fujimoto K, Maruyama T, Okuno K and Shindo K: Dose lateral lymph node dissection improve survival in rectal carcinoma? Examination of node metastases by the clearing method. J Am Coll Surg 184: 475-480, 1997.

19. Sato H, Maeda K, Maruta M, Matsumori K and Koide Y: Who can get the beneficial effect from lateral lymph node dissection for Dukes C rectal carcinoma below the peritoneal reflection? Dis Colon Rectum 49: 3-12, 2006. 